\title{
PARITY OF CLASS NUMBERS AND WITT EQUIVALENCE OF QUARTIC FIELDS
}

\author{
STANISLAV JAKUBEC, FRANTIŠEK MARKO, AND KAZIMIERZ SZYMICZEK
}

\begin{abstract}
We show that 27 out of the 29 Witt equivalence classes of quartic number fields can be represented by fields of class number 1. It is known that the remaining two classes contain solely fields of even class numbers. We show that these two classes can be represented by fields of class number 2 .
\end{abstract}

\section{INTRODUCTION}

A relationship between the Witt equivalence of quadratic number fields and the parity of the ideal class numbers has been noticed in [6]. There are seven Witt equivalence classes of quadratic number fields and it turns out that six classes contain some quadratic number fields with class number one, while the seventh class represented by the field $\mathbb{Q}(\sqrt{-17})$ consists exclusively of fields with even class numbers. As reported in [6], P.E. Conner offered a partial explanation for this phenomenon by pointing out that, for fields of arbitrary degree, the entire Witt equivalence class consists of fields with even class numbers provided some Witt equivalence invariants assume specific values. These invariants are the global level, $s(F)$, of the number field $F$ and the local dyadic levels, $s_{i}=s\left(F_{\mathfrak{p}_{i}}\right)$, where $F_{\mathfrak{p}_{i}}, i=1, \ldots, g$, are the dyadic completions of $F$. According to Conner, if $s(F)=2$ and $s_{1}=\cdots=s_{g}=1$, then the ideal class number of $F$ is even, and so also any number field $K$ which is Witt equivalent with $F$, has an even class number. Actually, Conner required the field $F$ to be totally complex with -1 a local square at every dyadic prime in $F$ but not a global square in $F$. These requirements are equivalent to $s(F)=2$ and $s_{1}=\cdots=s_{g}=1$, as can be seen by a straightforward application of the Hasse Local-to-Global Principle.

We do not know of any examples of Witt equivalence classes of number fields which consist exclusively of fields with even ideal class numbers and do not satisfy Conner's conditions. In this note we propose to show that all quartic Witt equivalence classes can be represented by fields with odd class numbers except for those satisfying Conner's conditions. Thus the situation for quartic fields is very much like that for quadratic fields. Our proof will supply an appropriate example of a quartic field in each of the 27 quartic Witt equivalence classes which do not satisfy Conner's conditions. A more conceptual proof would be desirable but it has escaped our attention.

Received by the editor August 2, 1994.

1991 Mathematics Subject Classification. Primary 11E81, 11 A07.

(c) 1995 American Mathematical Society 


\section{The CASE $s(F)=2$ AND $s_{1}=\cdots=s_{g}=1$}

For the convenience of the reader we recall the definition of the Witt equivalence invariant

$$
S(F)=\left(n, r, s, g ; n_{1}, \ldots, n_{g} ; s_{1}, \ldots, s_{g}\right)
$$

of a number field $F$ (see [6]). It consists of the following field invariants:

the degree $n$ of the field $F$ over the field $\mathbb{Q}$ of rational numbers, the number $r$ of real embeddings of $F$ into the field $\mathbb{C}$ of complex numbers, the global level $s=s(F)$ of the field $F$, the number $g$ of dyadic primes $\mathfrak{p}_{1}, \ldots, \mathfrak{p}_{g}$ of $F$, the local degrees $n_{i}=\left[F_{\mathfrak{p}_{i}}: \mathbb{Q}_{2}\right]$ of dyadic completions $F_{\mathfrak{p}_{i}}$ of $F$ over dyadic numbers $\mathbb{Q}_{2}$, and

the local levels $s_{i}=s\left(F_{\mathbf{p}_{i}}\right)$, where $i=1, \ldots, g$.

Here the level $s(K)$ of a field $K$ equals 0 if $K$ is formally real, otherwise it is the least number of summands in an expression of -1 as a sum of squares of elements from $K$. It is well known that the level $s(F)$ of a number field $F$ assumes only the values $0,1,2$ or 4 . The Witt equivalence invariant classifies completely algebraic number fields with respect to Witt equivalence (that is, up to isomorphism of their Witt rings of quadratic forms). See [5] and [6] for details and related references.

Now we will prove the following fact, first noticed by Conner.

Proposition. Let $F$ be a number field such that $s(F)=2$ and $s_{1}=\cdots=s_{g}=1$. Then the class number of $F$ is even, and every number field Witt equivalent to $F$ has also even class number.

Proof. Suppose the global and local levels satisfy the hypotheses and the ideal class number $h$ of the field $F$ is odd. Take a dyadic prime $q$ of $F$. Then $\mathfrak{q}^{h}=(a)$ is a principal ideal and $a$ is a nonsquare in $F$, since $h$ is odd. We claim that all Hilbert symbols $(-1, a)_{\mathfrak{p}}$ are trivial, that is,

$$
(-1, a)_{\mathfrak{p}}=1
$$

for all primes $\mathfrak{p}$ of $F$. For a dyadic prime $\mathfrak{p}$ this is obvious since -1 is a local square at $\mathfrak{p}$ (the local dyadic levels are all equal to 1 ). When $\mathfrak{p}$ is a finite nondyadic prime, then $a$ is a local unit at $\mathfrak{p}$ (by our choice of $a$ ), hence again the Hilbert symbol is trivial. Since $s=2$, there are no real infinite primes, and at complex infinite primes any Hilbert symbol is trivial. This proves our claim. However, -1 is not a global square, hence the nondegeneracy of the Hilbert symbol (combined with our claim) implies that $a$ is a global square, a contradiction. Hence $h$ must be even. If $K$ is any number field Witt equivalent to $F$, then $K$ and $F$ have the same Witt equivalence invariant. Hence, if $F$ satisfies Conner's conditions, so does $K$, and, as has been already proved, it has even class number.

We are concerned here with the classification of quartic number fields up to Witt equivalence so that $n=4$. First we want to single out those Witt equivalence invariants which satisfy Conner's requirements. Observe that, if the local dyadic degree $s_{i}=1$, then the corresponding local degree $n_{i}$ has to 
be even, and then use the fundamental equality $n=n_{1}+\cdots+n_{g}$ to conclude that there are exactly two possible values of $g$ giving $g=1, n_{1}=4$ and $g=2, n_{1}=n_{2}=2$. It follows that there are exactly two Witt equivalence classes of quartic number fields satisfying Conner's conditions (these are listed as types 6 and 17 in the Table (see next page)). By the Proposition, these two Witt equivalence classes do not contain any fields with odd ideal class numbers. Our intention is to show that each of the remaining classes contains at least one field with an odd class number. In fact, we show that one can always choose a representative with class number one.

\section{Commentaries to the TABle}

The values of $n, r, s, g, n_{i}, s_{i}$ in the table below form the Witt equivalence invariant $S(F)$ of the field $F=\mathbb{Q}(\alpha)$, where $\alpha$ is a zero of the polynomial $f(x)=x^{4}+a x^{3}+b x^{2}+c x+d$ with the coefficients $a, b, c, d$ given in the same row of the table.

The type is the Witt equivalence class determined by the corresponding value of $S(F)$.

Note that this is the same notation as in Table 1 of [4]. In that paper the Witt equivalence classes of quartic number fields have been described. See also [7], where the 26 classes containing 2-extensions are constructed in a systematic way.

The last column of the table gives the value $h$ of the class number of the field $F$. This has been determined with the aid of the results in [2].

The types 1-20 for which $g \leq 2$ are represented by fields $F$ of odd index (more precisely the generating element $\alpha$ has odd index in $F$ ). We have found the corresponding fields by applying Theorems 1-4 of [4].

For types 21-29 with $g=3$ or 4 , every field $F$ has an even index. The types 21-26 were covered with the use of Theorem 5 of [4] and types 27-29 were completed with the help of [1, Chapter IV, $\S 2$, Theorem 3 and Chapter VI, §3, Theorem 3] (see also [4]).

As a result of the information gathered for quadratic and quartic number fields, the following question, first asked by Conner, arises:

Question. If a number field $F$ has the level $s(F)$ different from 2 or at least one of the local dyadic levels $s_{i}$ is not equal to 1 , then is $F$ Witt equivalent to some number field with odd class number?

We have answered this question in the positive for quartic number fields, and by [6], the same holds for quadratic number fields. As to cubic number fields, they are all formally real so that $s=0$ and we should expect that every cubic number field is Witt equivalent to a field with odd class number. This is indeed the case. For Corollary (3.4) in [6] gives a list of representatives of the eight cubic Witt equivalence classes and seven of these are fields with odd class number, while the eighth, numbered II in [6], can be replaced with the field generated by a zero of the polynomial $x^{3}-3 x+4$, and this field has class number one. We owe this observation to A. Czogata ([3]).

However, in the general case, we are unable to answer the question, and more insight (rather than numerical data) is needed. 


\section{TABLE}

$\begin{array}{rrrrcccrrrrr}\text { type } & n & r & s & g & n_{i} & s_{i} & a & b & c & d & h \\ 1 . & 4 & 4 & 0 & 1 & 4 & 1 & 0 & -6 & -4 & 2 & 1 \\ 2 . & 4 & 4 & 0 & 1 & 4 & 2 & 0 & -4 & 0 & 2 & 1 \\ 3 . & 4 & 2 & 0 & 1 & 4 & 1 & -2 & -4 & 0 & 2 & 1 \\ 4 . & 4 & 2 & 0 & 1 & 4 & 2 & -1 & 0 & 2 & -1 & 1 \\ 5 . & 4 & 0 & 1 & 1 & 4 & 1 & 0 & -1 & 0 & 1 & 1 \\ 6 . & 4 & 0 & 2 & 1 & 4 & 1 & 0 & 18 & -60 & 50 & 2 \\ 7 . & 4 & 0 & 2 & 1 & 4 & 2 & -1 & -1 & 1 & 1 & 1 \\ 8 . & 4 & 4 & 0 & 2 & 1,3 & 4,4 & -1 & -4 & 1 & 2 & 1 \\ 9 . & 4 & 4 & 0 & 2 & 2,2 & 1,1 & -2 & -5 & 6 & 2 & 1 \\ 10 . & 4 & 4 & 0 & 2 & 2,2 & 1,2 & 0 & -5 & 0 & 2 & 1 \\ 11 . & 4 & 4 & 0 & 2 & 2,2 & 2,2 & -2 & -11 & -4 & 2 & 1 \\ 12 . & 4 & 2 & 0 & 2 & 1,3 & 4,4 & 0 & -1 & -3 & -1 & 1 \\ 13 . & 4 & 2 & 0 & 2 & 2,2 & 1,1 & -2 & -5 & -2 & 2 & 1 \\ 14 . & 4 & 2 & 0 & 2 & 2,2 & 1,2 & 0 & 1 & -2 & -2 & 1 \\ 15 . & 4 & 2 & 0 & 2 & 2,2 & 2,2 & -2 & 1 & 0 & -2 & 1 \\ 16 . & 4 & 0 & 1 & 2 & 2,2 & 1,1 & -2 & -1 & 2 & 2 & 1 \\ 17 . & 4 & 0 & 2 & 2 & 2,2 & 1,1 & -2 & -9 & 10 & 34 & 2 \\ 18 . & 4 & 0 & 2 & 2 & 2,2 & 1,2 & -2 & -13 & 14 & 58 & 1 \\ 19 . & 4 & 0 & 2 & 2 & 2,2 & 2,2 & 0 & -1 & 0 & 2 & 1 \\ 20 . & 4 & 0 & 4 & 2 & 1,3 & 4,4 & -1 & 2 & -1 & 2 & 1 \\ 21 . & 4 & 4 & 0 & 3 & 1,1,2 & 4,4,1 & -2 & -15 & -6 & 14 & 1 \\ 22 . & 4 & 4 & 0 & 3 & 1,1,2 & 4,4,2 & -2 & -4 & 5 & 2 & 1 \\ 23 . & 4 & 2 & 0 & 3 & 1,1,2 & 4,4,1 & -2 & -7 & 2 & -2 & 1 \\ 24 . & 4 & 2 & 0 & 3 & 1,1,2 & 4,4,2 & 0 & -2 & -1 & -2 & 1 \\ 25 . & 4 & 0 & 4 & 3 & 1,1,2 & 4,4,1 & -2 & 1 & 2 & 6 & 1 \\ 26 . & 4 & 0 & 4 & 3 & 1,1,2 & 4,4,2 & -2 & 2 & -1 & 2 & 1 \\ 27 . & 4 & 4 & 0 & 4 & 1,1,1,1 & 4,4,4,4 & -2 & -13 & 14 & 32 & 1 \\ 28 . & 4 & 2 & 0 & 4 & 1,1,1,1 & 4,4,4,4 & -2 & -5 & -2 & -8 & 1 \\ 29 . & 4 & 0 & 4 & 4 & 1,1,1,1 & 4,4,4,4 & -2 & -1 & 2 & 8 & 1\end{array}$

\section{ACKNOWLEDGMENT}

The authors would like to thank Professor M. Pohst for enabling them to use the tables of quartic number fields together with all the data cited in [2] and $\mathbf{M}$. Juentgen and S. Porubsky for their assistance in supplying these tables.

\section{BIBLIOGRAPHY}

1. Z. I. Borevič and I.R. Šafarevič, Number theory, 3rd rev. ed., "Nauka", Moscow, 1985. (Russian)

2. J. Buchmann, D. Ford, and M. Pohst, Enumeration of quartic fields of small discriminant, Math. Comp. 61 (1993), 873-879.

3. A. Czogala, Personal communication.

4. S. Jakubec and F. Marko, Witt equivalence classes of quartic number fields, Math. Comp. 58 (1992), 355-368. 
5. R. Perlis, K. Szymiczek, P.E. Conner, and R. Litherland, Matching Witts with global fields., Contemp. Math. 155 (1994), 365-387.

6. K. Szymiczek, Witt equivalence of global fields, Comm. Algebra 19 (1991), 1125-1149.

7. _ Witt equivalence of global fields. II: Relative quadratic extensions, Trans. Amer. Math. Soc. 343 (1994), 277-303.

Mathematical Institute, Slovak Academy of Sciences, Śtefánikova 49, 81473 BratisLAVA, SLOVAKIA

E-mail address: jakubec@savba.sk

Department of Mathematics and Statistics, Carleton University, 1125 Colonel By Drive, OtTawa, K1B 5B6, Canada

E-mail address: fmarko@math.carleton.ca

Institute of Mathematics, Silesian University, Bankowa 14, 40-007 Katowice, Poland E-mail address: szymiczek@gate .math.us.edu.pl 